The final, definitive version of this paper has been published in $<$ New Media $\&$ Society, Volume 11/Issue 8, 2009 by SAGE Publications Ltd, All rights reserved.

\title{
The question concerning (internet) time
}

\author{
Susan Leong, Teodor Mitew, Marta Celletti, Erika Pearson
}

\begin{abstract}
Spatial representations, metaphors and imaginaries (cyberspace, web pages) have been the mainstay of internet research for a long time. Instead of repeating these themes, this paper seeks to answer the question of how we might understand the concept of time in relation to internet research. After a brief excursus on the general history of the concept, this paper proposes three different approaches to the conceptualisation of internet time. The common thread underlying all the approaches is the notion of time as an assemblage of elements such as technical artefacts, social relations and metaphors. By drawing out time in this way, the paper addresses the challenge of thinking of internet time

as coexistence, a clash of fluxes, metaphors, lived experiences and assemblages. In other words, this paper proposes a way to articulate internet time as a multiplicity.
\end{abstract}

\section{Keywords}

actor-networks $\bullet$ assemblages $\bullet$ futures $\bullet$ internet $\bullet$ lived $\bullet$ metaphors $\bullet$ social $\bullet$ technology studies $\bullet$ temporal intensities $\bullet$ time 
The garden of forking paths is ... an infinite series of times, a growing, dizzying net of divergent, convergent and parallel times. (It is) a network of times which approach one another, fork, break off, or are unaware of one another. (Borges, 1970: 53)

Spatial representations, metaphors and imaginaries (cyberspace, web pages, virtual arena) have been the mainstay of internet research for a long time. This tendency has left internet research with a multitude of spatial concepts but very few concepts of internet time (Markham, 2003). Nevertheless, this remains a question crucial to the development of a deeper and more articulated understanding of the internet. This article considers the development of time as a general concept through history and proposes three possible approaches to the question of how we might understand and employ this concept in relation to internet research. Each of these approaches is enframed by different understandings of how technology and society relate to each other. While some of them dwell on the nature of the question of internet time, others explore probable solutions and negotiations. In other words, the deliberate diversity of the approaches outlined here is a calculated bid to refresh and reinvigorate discussion on this important question.

The first section briefly explores the history of the concept of time within the broad context of the Western philosophical tradition. It explores, in particular, three critical understandings of time: that of classical philosophy, the Kantian and the contemporary. The latter, as a developing and hence unstable paradigm, is constructed through a composite view of contemporary science and philosophy after the emergence of chaos theory. It suggests that the Deleuzian reconstruction of the historical significance of time might be one means to define the concept of time within a network of concepts and facilitate its inclusion in discussions of internet research on time.

The second section discusses the use of metaphors as tools of communication to develop consensual understandings in virtual environments. In particular, it highlights the effects that the metaphors brought online by participants have on future possibilities of the internet. In doing so, it draws attention to how the internet, as an intangible constructed social space, can be shaped by the different metaphors of time adopted by users (and researchers), which both enable and constrain certain decision pathways and outcomes.

The third section takes a wider approach, informed by the view that research on the social implications of the internet cannot be confined merely to its users because, as it contends, the internet's effects extend far beyond the immediate boundaries of those who use it. Therefore, this section advances an understanding of time in relation to the internet which is based on the notion of the lived, as articulated by Henri Lefebvre in his consideration of space. It suggests that such an application of the notion of lived time would 
better accommodate within its framework both users and non-users of the internet.

Finally, the last section suggests that the bifurcation of time into lived and instrumental temporalities occludes the multiplicity of timings involved in the internet assemblage, and prevents researchers from tracing the full depth of technical mediations. Instead, the section suggests that approaching network temporalities in their richness and depth requires tracing the modalities of technical objects. This approach has no use for the bifurcation between clock time and the time of humans, and instead concerns itself with measuring the intensity of timings. Timing, in turn, is always an inscription to a place, never a substitution from it; it is an additional layer we add to an always already lived reality of which techniques and machines are an integral part.

This article addresses the challenge of thinking internet time as a multiplicity of fluxes, metaphors, lived experiences and assemblages. The possibility of that multiplicity is explored in the following section, which aims to re-trace the main passages of the historical construction of the concept of time and to sketch a contemporary understanding of it, through the thought of Gilles Deleuze $(1994,2004)$. In this sense, this section acts as a background of the proposed approaches because it exposes their common roots and the general contemporary philosophical frameworks in which they reside.

\section{A BRIEF HISTORY OF THE CONCEPT OF TIME}

In pre-Socratic philosophy concepts are often embodied by divinities. Time as we commonly understand it is incarnate in Chronos or Aion, a god emerging from primordial Chaos to superintend the Ages. Although a very powerful divinity, Chronos represents mainly the quantitative nature of time. Accordingly, he is subordinate to history, ${ }^{1}$ that is, to the events that occur within time itself. Qualitative moments or 'special occurrences of time' are instead represented by a different god, Kairos.

In his interpretation of pre-Socratic philosophy, Plato understands time essentially as Chronos, and in particular either as 'the number' which measures the divine order, or as 'the calendar' which describes this order in a cyclic way. It is after Plato that Aristotle introduces a conceptual shift from numbering and the measurement of the cyclic movement of the stars, to the very movement of the stars (astrology and astronomy). Within both the Platonic and Aristotelian frameworks, time is 'order' rather than chaos (Deleuze, 2004).

Compared to classical philosophy, the philosophy of Kant represents a revolutionary approach to time. Within the Kantian framework of understanding, reality is a sequence of phenomena deployed in time. Reality is thus mirrored by the sequence of time's units. Accordingly, Kantian time is uni-dimensional, sequential and ordered. Implicit within Kant's 
re-conception of time are three major shifts. Firstly, from representing the order par excellence, time becomes with Kant 'something' that is ordered. Secondly, from referring to a cyclic, circular movement, time becomes a linear movement, where the beginning is god, or empty time, and the end is eternity, or the absence of time. Thirdly, rather than being subordinated to events happening in time, i.e. its quality, time is now what everything and everyone else is subordinate to (Kant, 1997[1788], 1998[1787]).

It is no coincidence that the Kantian linear conception of time is, essentially, the one first adopted by Newton and Newtonian science. Indeed, Kant is said to have been saturated with Newtonian physics (Whitehead, 1953). Both see time as linear, which has had important consequences for science and 'laboratory practice'. Since Newton, in fact, scientific experiments have been characterised by the comparison of the past with the future, performed in the present. In other words, laboratory time is conceived as a sequence of predictable ordered moments, a series that can be traced and retraced in both directions with the trickery of mathematics (Latour \& Woolgar, 1979).

Within this practice, conditions that cannot be observed in situ are 'recreated' in the laboratory. That is, past or future conditions are explored by moving the present experiment along the axis of a linear time. The latter operation, known as the calculation of the timeline of a dynamic system, is a mathematical calculation of classic dynamics that locks time into an infinite series of static frames, similar to movie-frames. One major constraint of such an operation is the absolute precision on which such calculations are based, a precision that requires the perfect control of every element of any such experiment (Prigogine \& Stengers, 1984).

However, the Newtonian conception of time has become untenable because a full investigation of unstable systems has evidenced the impossibility of total control. Thermodynamic systems, for example, have always resisted complete control and have been responsible for some of the major dilemmas of disorder. This is because thermodynamic systems sometimes undergo unpredictable and irreversible change in time, while losing more and more energy as they go. Dissipation and continuous change of status make precision almost impossible and undermine laboratory practice. In particular, they call for a re-conceiving of dynamics and its laws, that is, time and its management in science. Hence, through the study of thermodynamic systems, and more generally that of all systems tending towards entropy and disorder, the concept of time is being reshaped once again, to account for unstable conditions. This is why, metaphorically speaking, the concept of chaos has recently emerged with renewed significance from the irremediably fractured linear time of Kant. 
Nevertheless, the fracture of linear Kantian time actually pre-dates thermodynamics and occurs much earlier within philosophical discourse. The definition of the concept of time, as well as of chaos, is one of the major issues at stake in the so called 'science war' waged throughout the entire twentieth century. Probably the most critical point of this 'war' occurred when Henri Bergson failed to defend the cause of coexistence of the multiplicity of lived times (where true duration is understood as a qualitative multiplicity or time that is flowing (Bergson, 1910: 110) within real time. As Einstein put it, the intuitive coexistence of different durations in the same world was rejected by science and the very idea of a multiplicity of times was labelled as incompetence (Prigogine \& Stengers, 1984).

Although Einstein and Poincare, in different ways and with different agendas, had shaken the foundations of Newtonian mechanics with the theory of relativity, the concept of time within it advances only by the first of the many steps Bergson anticipated (Galison, 2003). The theory of relativity, in fact, transforms the Newtonian conception of a single uniform and unidirectional time in a relativist conception of multiple times relative to multiple moving observers. In other words, it works through a more efficient, synchronised conception of time as a clock time, efficiently supporting the nascent globalisation of market and labour belonging to modernity. The early work of Poincare, instead, centered on the principles of chaos theory rather than the modernist vision of clock time, later allowed a wider understanding of thermodynamics and complexity.

However, the confrontation between natural sciences and human sciences is still the locus where the re-conception of time remains trapped and strives to emerge; only recently have some convergent analyses been developed, which offer opportunity for discussion and reflection. Most of them spring from the redefinition of previously closed concepts such as chaos, duration, speed and synchronicity. In the realm of science, for example, the concept of chaos and the focus on complex systems head a move towards the rearrangement of the role of time in scientific research practice (Nikolis \& Prigogine, 1989). Analogously, philosophy, and in particular French philosophy, has recuperated the intuition of Bergson on duration to create an intellectual framework capable of accommodating a different idea of time (Guattari, 1995).

Within a convergent perspective of science and philosophy, Deleuze (1994) posits a re-conception of time as constructed through the historical development of the concept itself. According to him, it was the conception of cyclic time that led to the establishment of habits. That is, of passive syntheses of moments, as they are understood within a cyclical repetition (day/night, seasons, birth/death). These same habits make sense of time as a continually living present. Linear time, in contrast, makes sense of time mainly as memories. That is, the active syntheses of moments which never 
repeat themselves so that they never become habits. These same memories therefore regard time as a past that has never been present. Deleuze associates both habits and memories with the characteristics belonging to the old Greek god, Chronos.

In their stead, Deleuze proposes a time conceived as a future. In other words, instead of the repetition of the identical, typical of cyclic time, or the absence of repetition proper of linear time, he suggests that contemporary understandings of time embrace the repetition of the differences. This repetition is intended as the representation of moments that are never identical to the ones to be represented, precisely because once re-acted or re-enacted they become different from the original. The repetition of differences makes sense of time as a future, as a becoming. Deleuze envisages this form of time as the re-emergence of the qualities of the lesser known Greek divinity Kairos (the instant, the event). Such a re-conceptualisation would be most powerful as it has the advantage of repeating the past as a form of present but in a different time and in an undetermined way, outside the sequence of linear time. In this sense, it resembles the characteristics of chaos as it stands for the unpredictable unfolding of the present, an unfolding which, signified by the internet, stands for a cohabitation of difference.

\section{METAPHORS OF INTERNET TIME AND FUTURE STUDIES}

Time, imagined as a multiplicity of differences, is difficult to grasp. Metaphors are one of the main strategies that can be employed to grasp such abstract notions. Spatial and social relations that occur on the internet are also defined, at least in part, by the consensual metaphors used by the participants. Metaphors are of interest not only in terms of how they work to define and redefine virtual spaces and interactions, but also for what they reveal about how the participants' mental models or paradigms promote or deny certain choices. Metaphors offer a way of understanding the processes that lead to choices that participants make within the context of their online interactions, and that affect their future possibilities. It is this use of metaphor, as a way of opening up and exploring potential futures issues, which is of particular interest in this discussion.

Metaphors refer not only to the descriptors and language used (e.g. 'time flies'), but also betray how users think about and engage with the system (Cornish, 2004). Elements that fit the metaphoric system are seen as 'obvious'; elements that do not can be marginalised within the discussion. For example, the early metaphor of internet as a 'superhighway' privileged linear spatial models of the virtual over more complex three-dimensional concepts of space and place online. Similarly, but on a somewhat grander scale, the Kantian and scientific conceptions of time discussed earlier continue to shape expectations and paradigms of time, and are privileged as rational, logical 
and 'matter of fact' in some cultures. Futures studies challenges such acts of privileging by seeking to deconstruct the assumptions and limitations of existing metaphors, and by considering how alternative metaphors may alter perceptions of what may be possible (Inayatullah, 2005).

Within a singular cultural context, metaphors of time can appear ubiquitous - for example, some cultures often describe time as a river, a unidirectional flow from a past to a future. But there is no singular ubiquitous 'internet culture' as such. Rather, users from different backgrounds converge and interact to various extents with users from other contexts. These different users bring with them different metaphors and understandings of time. Inayatullah (2005) notes that some of these include metaphors of degeneration and stagnation, metaphors of time as timelessness with no beginning or end, cosmological time, or time as a lifecycle where each ending is another beginning. Each of these metaphors brings with it assumptions and framings of the link between past, present and future. For example, time as degeneration would seem to suggest a sense of inevitable decline, whereas time as a lifecycle brings with it a framework of constant renewal. These different metaphoric framings therefore shape how users engage with ideas of the future, and how they pursue desirable futures or let the future 'wash over them.'

Leaving aside for one moment the highly problematic issue of how to approach and develop understandings of the multiple metaphors of time online, this discussion will first turn to look briefly at how these different metaphors interact or suggest different futures potentials.

Futures studies is interested in metaphors for a number of reasons. Firstly, metaphors betray the paradigms and mental models their users possess (Dator, 1997; Rycroft, 2006; Wyatt, 2004). This suggests what may be considered plausible (and preferable) to these users as they consider their options and make choices which affect the future of the system. Macrohistory approaches to futures questions continue this idea, and analyse metaphors in order to challenge dominant paradigms of time, space and culture (Inayatullah, 1998, 2005). Indeed, understanding and working with metaphors constitutes a key element to more recent third wave futures research. These newer research approaches seek to not just passively forecast futures possibilities, but to actively draw attention to such constraining paradigms and to suggest other options and other ways of understanding or negotiating the constraints these paradigms impose on users (Inayatullah, 2005; Slaughter, 2004).

This brings us back to the metaphors of time online. Whilst there is not sufficient room to develop an analysis of all metaphors of time that can be found on the internet, it is still illustrative to look at some examples to demonstrate how futures studies may work with such metaphoric understandings to explore future possibilities for a transnational system such as the internet. 
Firstly, it needs to be emphasised that metaphors are representative of underlying cultural, social and political values (Ihsan, Inayatullah \& Obijiofor, 1995). They do not occur in a vacuum, and as such there is an investment in the underlying assumptions that support such metaphors. For example, Asian metaphors of time include the metaphor of the tree, with roots in the past and many branching options into the future (Inayatullah, 2005). This metaphor, different from Western ideas of time as river, also reflects values such as respect for the past and cultural predecessors. On a system such as the internet, which is hyped as a flat, egalitarian hierarchy (though that itself is challengeable), it cannot be assumed that one metaphor, and its related sets of values and priorities, should automatically become dominant. One consequence of making explicit the underlying values of metaphors is the revelation and indeed, awareness of, the political aspects to understandings of internet and time.

This is not to say that metaphors are empty or meaningless in themselves. Metaphors are replicated and reinforced through language and the processes of communication, and it is within this act of communication that meaning is constructed and, more importantly for futures studies, reconstructed (Ihsan et al., 1995). Stevenson (1998) goes as far as to argue that this communication across cultures may lead to new negotiations regarding metaphors and the assumptions that support them, with subsequent alterations in terms of the perceived range of choices available. In other words, by comparing personal or cultural metaphors in a multicultural space such as is potentially possible online, the political values will become clearer, and will lead to a renegotiation of metaphors and their underlying values. It is still to be seen whether such renegotiation of metaphors will occur in any widespread fashion online, or whether separate metaphoric frameworks will exist alongside one another in cybernetic cultural enclaves, such as can be seen already with the different language groups online.

Competing metaphors of time can also have a strong effect on how actively participants pursue different futures outcomes (Ambos-Spies, Home \& Schöning, 1993; Waldrop, 1992). For example, compare two metaphors already cited: time as a river and time as a tree. Time as a river carries with it connotations of passivity: time flows on, time passes. Time, in this metaphoric framework, will move from a source, a singular beginning to an unspecified singular end, no matter what. Conversely, the metaphor of time as a tree is fixed, literally rooted and grounded in a past, with a panoply of possibilities 'branching out' above. There is the implication of options, of engagement, of active choice within the range of potentials supported by the existing past, which itself is multi-nodal and drawn in from more than one source. These two metaphors of time yield two frameworks which locate the past, the future and the participant in two contradictory positions. 
Online, this issue is magnified as there are as many metaphors as there are cultures logged in. Through discussing and deconstructing the assumptions and constraints of these metaphors, discontinuities between paradigms and approaches to understanding the internet may be uncovered, and new possibilities or new ways to approach choice and change might reveal previously unconsidered futures potentials.

\section{LIVED TIME}

A number of metaphors have long been dominant to understandings of how the internet and time relate, and they continue to overshadow others for a number of reasons. Most of these metaphors are conditioned by the imaginings and experiences of internet users. One of the key problems facing understandings of time in research, therefore, stems from the central view that any effect the internet may have is experienced only by those who use the medium. Such a view is reinforced, and indeed implicitly endorsed, when non-users are omitted altogether in studies of the social implications of the internet. However, as Wyatt (2003) argues, non-users do matter and developments in the relationship between the internet and society cannot help but impinge not only on those who use the internet but must also bear consequences for the wider society who both constitute and share in the experience of everyday life.

One task confronting us, then, is the formation of a wider framework able to accommodate broader, more inclusive contexts. This section does so by drawing on the work of two theorists, Lefebvre and Durkheim, who each offer an understanding of time incorporating the social and the everyday. To begin, we briefly explore the possibilities of extending the notion of 'the lived' developed by Lefebvre (1991) in thinking about space, to time. Although Lefebvre's work was formulated primarily through an exploration of spatial issues, the notion of the lived would profit from being applied to thinking about time. This notion allows us to conduct wider, more inclusive studies of the social implications of a technology. This is because lived time (and space) is both a product and a pre-condition of social practices, and there can be few more effective ways to study the social implications of a technology than through the social practices that ensue from it.

The lived is described by Lefebvre as the social construct that dwells halfway between the abstract and concrete constructs (1991). For him, it is socially formed and the 'materialization of social being' (1991: 102). In The Production of Space, he lays out a spatial triad consisting of abstract, concrete and lived space (1991:33, 38-39). Within this triad, abstract space is the representation of space and hence, the mentally conceived space of knowledge, logic, signs and codes. The 'instrumental space' (Elden, 2001: 815 ) of architects, engineers and planners, abstract space is largely symbolic in 
nature. Concrete space, in contrast, is the physical perception of space, usually encapsulated in spatial practice. As practice, concrete space implies both habit and cohesion in understandings of how space is used or put to work. Concrete space is thus the space of production. ${ }^{2}$ The interpenetration of abstract space and concrete space, according to Lefebvre, is what constitutes lived space. Lived space is the space of its inhabitants, it 'overlays physical space, making symbolic use of its objects' (Lefebvre, 1991: 39). It is socially constructed and hence, the space of representation. ${ }^{3}$

However, Lefebvre cautions against the simple equation of lived space with space that has been socialised (1991: 190), ${ }^{4}$ as such an equation of the form of space with its function is a simplistic, reductionist understanding of how space works (pp. 33-34). It is important to realise that the intent behind the triads of abstract - lived - concrete or conceived - lived - perceived is not to cut space up into its component parts. For implicit within the interpretation of space (and time) as a triad is the assumption that whatever space comes to mean at any one point is a product of a transformative process. The nature of this process is intrinsically dynamic and is thus always open to the vagaries, complexities and contradictions of social life and its associated tensions. Hence, Lefebvre's triad is an articulation of the different ways in which both time and space can be grasped as lived, with all the potential of assembly (and accumulation) at or around a single point (p. 101). The joining of the notion of the lived to that of time and space emphasises the role of social praxis, for they embody a dialectics of possibilities that can be realised in an infinite number of ways through social acts and practices.

In The Elementary Forms of the Religious Life (1976[1912]), Durkheim asserts that it is the 'rhythm of social life ... which is at the basis of the category of time' (p. 440). According to him, at the core of intellectual life is a 'framework of intelligence' (p. 9) made up of essential, universal ideas including those of time and space. This is an abstract, impersonal framework and the universal ideas it consists of belong to and act as guidelines for everyone. Within such an understanding, time is not individually but generally defined, 'objectively thought of by everybody in a single civilization' (p. 11). In other words, the elements of the framework themselves sit above (or beyond) the various methods of differentiating time (clock, calendar). Durkheim argues that since all the people within a single civilisation harbour the same notions of these core ideas it stands to reason that the sources of these ideas are collective and have a social origin (p. 11).

According to Durkheim, all logical thought or collective concepts, including the social concepts of time and space (1976[1912]: 441-2), exist as they do because they are born of the unique intelligence of humankind, of 'collective consciousness' (p. 444). Instead of intuition, he attributes the concepts of time and space to this external intelligence, which though it 
'bears the mark of no particular mind' is 'where all others meet each other' (p. 434). Through his insistence that the basis of social time is collective, and hence social (p. 9), Durkheim can be seen to invert our understanding of time from both a Kantian and a Bergsonian point of view. Indeed, for him, the Kantian notions of transcendental time and space and the Bergsonian ideas of duration and flow cannot but also be sets of interpretations (representations) that society puts collectively to time and space (pp. 16-17).

Understood after the manner of Durkheim, the notion of social time privileges the social over the individual. However, as even Lefebvre had to reiterate in his later work, 'social time is itself a social product' (2004: 73-74). Concerning time at least, Durkheim and Lefebvre concur: our experiences of time spring from our experiences of the social. If our task is to explicate on the social implications the internet might have on our everyday life, we would do well to begin with an examination of the collective experience of lived time.

\section{ASSEMBLED TIME}

Nevertheless, to examine a given collective experience of internet time, one first needs to trace just who and/or what comprises the collective, and therefore whose time is being examined. Accordingly, a tracing of the effects of time, and internet time in particular, should arguably start with a tracing of both the actors comprising the collectives and the assemblages producing the time.

However, the task of tracing is often complicated by a tendency to rely on a separation between social and technical strata, or to view the technical as somehow secondary to and detrimental to the social proper. This tendency is made explicit in the work of Manuel Castells, for whom 'timeless time' and the 'space of flows' are the characteristics of the 'network society' (Castells, 2000: 13). In this understanding the logic of information networks constitutes an entirely new paradigm (Castells, 2004) enframing (in the Heideggerian sense) the social. 'Lived time' is 'annihilated' into an instrumental 'timeless time' and space is de-linked from its geographic locatedness into a 'space of flows.'

Castells' approach is symptomatic of what could be described as the bifurcation of network temporality. Here time is approached either as a loss propelled by the instrumental logic of network technology, or as an obliteration of 'embodied subjectivities' and their local social relations. This position stems from a particular understanding of technology and its effects on time and space. Accordingly, time as a product of a technique always remains secondary to a more primary ontological or social reality. Simultaneously, when technical time is seen as a deadening of 'lived' time, technical objects assembled around it are excluded.

At the basis of this bifurcation is the belief that in the modern world there exist two essentially opposite types of temporality. One is a human, social 
and lived temporality, the idea of which, when stretched, vaguely resembles the ancient Greek concept of Kairos. The other in turn is an anachronistic projection which sees the emergence, sometime in the middle centuries, of a radically inhuman and instrumental form of temporality, which, since then, has gradually established itself as the primary temporality of the modern world. This is the temporality of the clock, which reads like an extreme remake of the position occupied in ancient Greece by Chronos.

The key issue in this bifurcation is the confusion between time and the measurement of time (Serres \& Latour, 1995: 60). Clock time is seen here as an artificial addendum to a primary social and/or natural temporality. Accordingly, clocks are believed to create a specific clock time of pure instrumentality, obfuscating temporality as emergence and substituting it with a temporality of orderings (Hassan, 2003, 2005). As Adrian Mackenzie demonstrates, the proponents of this position 'reduce temporal complexities ... to intervals and orderings that are inimical to human lifeworld structures' (Mackenzie, 2005: 2).

However, one cannot start answering questions about networks, without accounting for technical mediations as intermediaries constituting 'a regime of timings and spacings from which society and nature, time and space unfold' (Mackenzie, 2002: 95). In such a regime, temporality is always a measurement against a surface (Elias, 1992). For example, in Euler's famous solution to the Konigsberg bridge problem, time is flattened into space and all possible nodal links, existing at different points of time, are visible at once, as different surfaces. Euler's model places networks in a space of duration; when flattened into space, the entirety of a time can be visible in the present. Such a construct does not, however, form an ontological totality somehow separate from 'lived' embodied time/space. The effect of seeing everything from all sides appears 'because we're inside a room in which the illusion is mastered, and not outside' (Latour \& Hermant, 2006).

In place of temporal homogeneity, the spatial expansion of connectivities confronts us with an ever-expanding multiplicity of temporal events. The challenge in approaching this temporality is in the heterogeneity of the actors appearing in the assemblage. ${ }^{5}$ As Bruno Latour argues in an influential essay on formalism and time (1997), the difference between temporalities does not lie in an illusionary bifurcation between clock time and lived time, but in the intensities of actors' timings. These intensities are a function of the relation between the two simultaneous vectors of transportation and transformation.

Latour illustrates this relation with the example of two travellers, one sitting comfortably in a bullet train, the other hacking her way through a jungle. Both travellers move, yet they exist in radically different spaces and times. According to Latour, that is because while the first is transported without any visible transformation, that being in turn delegated and handled 
by a large support network of rail institutions, electrical power stations, technicians and machinery, the second traveller undergoes transformations with every step. While she has to undergo a series of transforming trials enforced by the jungle, the former traveller can revel in the effects of a seamless flow of speed.

What differentiates the flow of this traveller's movement from the discrete spacings of the jungle-explorer is not some fundamental difference between the techniques employed by the former and the living body of the latter, but the intensity, the ratio of transformation-to-transportation resulting from their movements. Timings and spacings appear out of these intensities, and not the other way around.

The effect of speed is accordingly the result of how much transformation an object undergoes during the trial of transportation. If the network transporting it is stable enough and allows someone or something else to transform instead, then the effect of seamless transportation without transformation occurs. The speed and seamlessness of internet flow is precisely such an effect. The idea of a speed inherent in technology, in some supposed instrumental logic, is therefore just an unfortunate illusion borne out of a bifurcated view of time and often ignorance of the way technical networks operate. $^{6}$

Instead of one homogenous global network time, this understanding reveals an assembly of timings, exemplified in this very article, edited through an online collaboration. The timing of this document, for now residing on an anonymous-to-us internet server, is an assembly of complexities. The fact that we see it as a more or less stable and homogeneous space-time is due to the intensity of the relation between transformation and transportation. If the document is stable and does not change more than we ask it to, it is because something else undergoes the necessary transformations thus allowing us to experience the effect of homogeneity.

In other words, instead of an isochronous network flow, a 'timeless time,' we should approach network temporality as a multiplicity of times derived from relations between different elements (Latour, 1997). The bifurcated projection would never even suspect the existence of this relation, for it sees only the dead instrumentality of clock time and the rich, lived time of the social. But, returning back to our as yet ephemeral online text, which part of it is instrumental and which social? How can we account for the lived time of four collaborating humans and the equally lived time of many more collaborating machines if we render our searching eyes oblivious to the complexity of the networks involved in supporting this collaboration? 'It is the sorting that makes the times, not the times that make the sorting' (Latour, 1993: 76). 
What makes this understanding even more compelling is the fact that on the internet the object, its components, and any commentary on that object 'exist contemporaneously and conceptually in the same place/non-place of the network' (Lunenfeld, 2005: 95). Taking into account this reality, and the 'spill-over' of the internet onto the everyday, requires us to dig into the temporality of internet data 'as if all the past were still present' (Latour, 1989).

That is because the entire assemblage of constantly re-deployed technical mediations holds together not one isochrony but a multitude of timings. If there appears the effect of a totality, 'it is obtained through a process of summing up, itself localized and perpetually restarted, whose course can be tracked' (Latour \& Hermant, 2006: 51). In other words, the effect of a local homogeneity of time is a constantly upheld event, behind which works a multitude of actors. Temporality, technical network or not, is perpetually enmeshed with a topology. A good example of multiple temporalities can be found in the proliferation of timings within information networks. These involve 'seek time, run time, read time, access time, available time, real time, polynomial time, time division, time slicing, time sharing, time complexity, write time, processor time, hold time, execution time, compilation time, and cycle time' (Mackenzie, 2005:2). The list could go on into the ever increasing complexity of database timings, wireless frequencies, signal-tonoise ratios, the timings of routing tables and so on and so forth. These timings, black-boxed within the network whose intermediaries they are, are invisible to us, unless of course they metamorphose from intermediaries to mediators. Their effect is a stable convention of timings, a series of 'temporal displacements' (Grosz, 2001: 88-89) and enfoldings through whose 'interstices' (Mackenzie, 2005: 3) percolates what we can only perceive as the flow of time.

Therefore, the challenge internet time poses is that of measuring an alwaysconstructed and composite collective intensity, that is, investigating who and what comprises the collective. This investigation is not dissimilar to an archaeology of temporal coexistences (Shanks, 1998). Most importantly, such an approach involves taking risks with time, because it brings forth objects as mediators. To view network temporality as the time of Kairos, of the event, is to involve intermediaries, techniques and unknown chains of stakeholders in a motion allowing its author to withdraw and testifying in her place (Stengers, 2000). Contrary to obliterating time, this motion creates a multiplicity of times.

The question concerning network temporality is therefore in many ways a function of the constantly reappearing search for a time-space existing in opposition to the instrumental. The internet, in its perceived isotopy and isochrony is just the latest assemblage to be suspected of 'pure' instrumentality somehow denying 'lived' experience. However, there is no point in looking 
to isolate a non-instrumental aspect of time. The issue is rather that of addressing the 'modalities of instrumentality' (Stiegler, 1998: 206) as such. In other words, the time of information networks is assembled, upheld and unfolded with particular local intensities, and it is these intensities that need to be explored, not the illusionary dialectic of instrumental and 'lived.' For to construct such a dialectic one has to obliterate an entire multitude of intermediaries upholding both the ephemeral instrumental and the event that wants to be thought as 'lived.'

\section{CONCLUSION}

This paper has sought to problematise ways of approaching and understanding the challenge of thinking about internet time. The presented approaches form a deliberately heterogeneous argument aimed at exploring paths for future research of network temporalities.

As the background discussion indicated, time as a philosophical concept has an extensive history. Different philosophical approaches redefined time as a series of events generating in turn a culture of habits, memories, passing time and laboratory practice. Today, the concept of time is able to represent the repetition of difference through an enactment of the present in a future virtual context of representation. This difference creates spaces in which multiple (parallel) perspectives of time can develop. This is exemplified by the differing metaphors of time that can be identified within transnational, multicultural and multilingual spaces such as the internet. Different ways of talking about, understanding and framing notions of time - the imaginaries of time - can have ramifications for how researchers approach and discuss this inchoate area of study. In order to account for the growing social and cultural diversity of internet users and participants, discussions of time need to acknowledge a diversity of understandings of time.

This diversity must also be acknowledged when considering that the lived experience of time is also a thoroughly social one. How we relate time to internet filters through into how we negotiate the daily confrontations between self, society and the technologies they encounter. The possibilities of applying the notion of the lived, as the 'materialization of social being' (Lefebvre, 1991: 102), to how we might approach the question of internet time is one ripe for further exploration.

Viewing objects and technical assemblages as active participants and intermediaries in the production of time and space opens the possibility of viewing temporality as perpetually enmeshed with a topology that has to be traced, studied and engaged. Moreover, by studying the logistics of assembled time we discover richness and depth where there was only supposed homogeneity. 
The common thread underlying all the approaches to internet time raised here is, as mentioned before, the notion of time as an assemblage. Furthermore, the authors argue that both the understandings of time in relation to the internet, and internet time itself, are coming out of a constellation of elements - technical artefacts, social relations and metaphors all playing their part in assembling, understanding, appropriating and engaging time. Woven throughout this is the idea that time is not and cannot be a homogeneous totality, external and unchanging. Rather, time is viewed as manufactured alongside all other elements that constitute that assemblage.

By drawing out and discussing time as an assemblage, a product of the intersection of various elements within a complex system such as the internet, this paper manifests the necessity, for internet research discourse, of conceptualizations capable of engaging such multiplicities either in a metaphorical, social or almost archaeological manner. It is anticipated that in drawing attention to this neglected area of internet scholarship, a more nuanced and detailed approach to time and the internet can be developed.

As the title already suggests, this article aimed to reinvigorate the conceptual approaches to internet time, and argue for a new set of questions concerning that temporality. The deliberate diversity of the conceptual approaches outlined in the article provides a setting for cross-pollination between otherwise disparate theoretical positions on time, and offers a comparative tool-kit of temporal conceptualizations for internet researchers looking to develop new methodologies for studying network temporalities.

Notes

1 The subordination of Chronos is justified by the fact that he is considered responsible only for quantifying time, measuring periods and seasons and therefore monitoring the repetitive, cyclic movement of the skies, but is not accountable for what time unfolds, for the quality of time.

2 This article uses Shields' (1999: 161) translation of Lefebvre's term (l'espace vécu) as 'spaces of representations.'

3 Lefebvre's interchangeable usage of the terms 'social space' and 'lived space' is continued here, with a preference for the latter term. The same applies to Durkheim's usage of social time.

4 Silverstone (1993) makes a similar point about the socially constructed nature of time, using the family unit and its daily activities as the locus.

5 For example, consider Beatriz da Costa's Pigeonblog Project: http://www.pigeonblog. mapyourcity.net/

6 For an interesting discussion of technology and mobility see Sheller, 2004; Sheller \& Urry, 2006; and Peters, 2006.

\section{References}

Ambos-Spies, K., S. Home and U. Schöning, (eds.) (1993) Complexity Theory: Current Research. Cambridge: Cambridge University Press. 
Bergson, H. (1910) Time and Free Will: An Essay on the Immediate Data of Consciousness (trans. F.L. Pogson). New York: Macmillan.

Borges, J.L. (1970) Labyrinths: Selected Stories and Other Writings. Harmondsworth: Penguin Books.

Castells, M. (2000) 'Materials for an Explanatory Theory of the Network Society', British Journal of Sociology 51(1): 5-24.

Castells, M. (2004) 'Informationalism, Networks, and the Network Society: A Theoretical Blueprint', in M. Castells (ed.) The Network Society: A Cross-cultural Perspective, pp. 3-48. Cheltenham: Edward Elgar.

Cornish, E. (2004) Futuring: The Exploration of the Future. Bethesda: World Future Society.

Dator, J.A. (1997) 'Futures Studies and Sustainable Community Development', in K. Yamaguchi (ed.) Sustainable Global Communities in the Information Age: Visions from Future Studies. Westport: Praeger.

Deleuze, G. (1994) Difference and Repetition (trans. P. Patton). New York: Columbia University Press.

Deleuze, G. (2004) Fuori dai Cardini del Tempo: Lezioni su Kant. Milan: Associazione Culturale Mimesis.

Durkheim, E. (1976[1912]) The Elementary Forms of the Religious Life (trans. J. Swain, 2nd edn). London: Allen and Unwin.

Elden, S. (2001) 'Politics, Philosophy, Geography: Henri Lefebvre in Recent AngloAmerican Scholarship', Antipode 33(5): 809-825.

Elias, N. (1992) Time: An Essay. Oxford: Blackwell.

Galison, P. (2003) Einstein's Clocks, Poincare's Maps: Empires of Time. New York: W.W. Norton \& Company.

Grosz, E. (2001) Architecture from the Outside: Essays on Virtual and Real Space. Cambridge, MA: MIT Press.

Guattari, F. (1995) Chaosmosis: An Ethico-Aesthetic Paradigm (trans. P. Bains and J. Pefanis). Bloomington: Indiana University Press.

Hassan, R. (2003) The Chronoscopic Society: Globalization, Time and Knowledge in the Network Economy. New York: Lang.

Hassan, R. (2005) 'Timescapes of the Network Society', Fast Capitalism 1(1), URL (consulted June 2007): http://www.uta.edu/huma/agger/fastcapitalism/1_1/hassan. html

Ihsan, S., S. Inayatullah and L. Obijiofor (1995) 'The Futures of Communication', Futures 27(8): 897-903.

Inayatullah, S. (1998) 'Macrohistory and Futures Studies', Futures 30(5): 381-394.

Inayatullah, S. (2005) Questioning the Future: Methods and Tools for Organizational and Societal Transformation. Taipei: Tamkang University Press.

Kant, I. (1997[1788]) Critique of Practical Reason (trans. M. Gregor). Cambridge: Cambridge University Press.

Kant, I. (1998[1787]) Critique of Pure Reason (trans. P. Guyer and A.W. Wood). Cambridge: Cambridge University Press.

Latour, B. (1989) 'The Enlightenment Without the Critique: A Word On Michel Serres' Philosophy', in A.P. Griffiths (ed.), Contemporary French Philosophy, pp. 83-98. Cambridge: Cambridge University Press.

Latour, B. (1993) We Have Never Been Modern (trans. C. Porter). Cambridge, MA: Harvard University Press.

Latour, B. (1997) 'Trains of Thought: Piaget, Formalism and the Fifth Dimension', Common Knowledge 6(3): 170-191. 
Latour, B. and E. Hermant (2006) 'Paris: Invisible City', URL (consulted July 2006): http://www.ensmp.fr/ latour/virtual/

Latour, B. and S. Woolgar (1979) Laboratory Life: the Social Construction of Scientific Facts. Beverly Hills: S(age-Publications.

Lefebvre, H. (1991) The Production of Space (trans. D. Nicholson-Smith). Oxford: Blackwell.

Lefebvre, H. (2004). Rhythmanalysis: Space, Time and Everyday Life (trans. S. Elden and G. Moore). London; New York: Continuum.

Lunenfeld, P. (2005) User: InfoTechnoDemo. Cambridge, MA: MIT Press.

Mackenzie, A. (2002) Transductions: Bodies and Machines at Speed. London: Continuum.

Mackenzie, A. (2005) 'Protocols and the Irreducible Traces of Embodiment: the Viterbi Algorithm and the Mosaic of Machine Time', URL (consulted May 2007): http:// www.lancs.ac.uk/staff/mackenza/papers/Mackenzie-algorithmic-time.pdf

Markham, A.N. (2003) 'Metaphors Reflecting and Shaping the Reality of the Internet: Tool, Place, Way of Being', paper presented at the Association of Internet Researchers Conference, Toronto, Canada, 16-19 October.

Nicolis, G. and I. Prigogine (1989) Exploring Complexity. Munich: GmbH \& Co.

Peters, P. (2006) Time, Innovation and Mobilities: Travels in Technological Cultures. New York: Routledge.

Prigogine, I. and I. Stengers (1984) Order Out of Chaos: Man's New Dialogue with Nature. New York: Bantam Books.

Rycroft, R.W. (2006) 'Time and Technological Innovation: Implications for Public Policy', Technology in Society 28(3): 281-301.

Serres, M. and B. Latour (1995) Conversations on Science, Culture, and Time (trans. R. Lapidus). Ann Arbor: University of Michigan Press.

Shanks, M. (1998) 'The Life of an Artifact', URL (consulted September 2006): http://traumwerk.stanford.edu/ mshanks/traumwerk/index.php/The $\% 201$ ife $\% 20$ of $\%$ 20 an\%20artifact

Sheller, M. (2004) 'Mobile Publics: Beyond the Network Perspective', Environment and Planning D: Society and Space 22(1):39-52.

Sheller, M. and J. Urry (2006) Mobile Technologies of the City. London: Routledge.

Shields, R. (1999) Lefebvre, Love, and Struggle: Spatial Dialectics. London: Routledge.

Silverstone, R. (1993) 'Time, Information and Communication Technologies and the Household', Time \& Society 2(3): 283-311.

Slaughter, R.A. (2004) Futures Beyond Dystopia: Creating Social Foresight. London: Routledge Falmer.

Stengers, I. (2000) The Invention of Modern Science (trans. D.W. Smith). Minneapolis: University of Minnesota Press.

Stevenson, T. (1998) 'Netweaving Alternative Futures: Information Technocracy or Communicative Community?', Futures 30(2/3): 189-198.

Stiegler, B. (1998) Technics and Time, 1: The Fault of Epimetheus (trans. R. Beardsworth and G. Collins). Stanford, CA.: Stanford University Press.

Waldrop, M.M. (1992) Complexity: The Emerging Science at the Edge of Order and Chaos. New York: Touchstone Publishers.

Whitehead, A. N. (1953) Science and the Modern World. New York: The Free Press.

Wyatt, S. (2003) 'Non-Users Also Matter: The Construction of User and Non-Users of the Internet', in N. Oudshoorn and T. Pinch (eds) How Users Matter: The CoConstruction of Users and Technologies, pp. 67-80. Cambridge, MA: MIT Press.

Wyatt, S. (2004) 'Danger! Metaphors at Work in Economics, Geophysiology, and the Internet', Science, Technology, \& Human Values 29(2): 242-261 
Leong et al.: The question concerning (internet) time 